\title{
Rutin Prevents High Glucose-Induced Renal Glomerular Endothelial Hyperpermeability by Inhibiting the ROS/Rhoa/ROCK Signaling Pathway
}

Authors

Affiliation
Xiaoming Wang, Xiaohong Zhao, Ting Feng, Gang Jin, Zhenjiang Li

Department of Nephrology, Shaanxi Provincial People's Hospital, Xi'an, Shaanxi 710068, P. R. China

Key words
rutin
high glucose
renal glomerular endothelial
permeability
RhoA/ROCK
Nrf2/ROS

received April 15, 2016 revised June 1,2016 accepted June 15, 2016

\section{Bibliography}

Dol http://dx.doi.org/ 10.1055/s-0042-110859 Published online August 23, 2016

Planta Med 2016; 82: 1252-1257 @ Georg Thieme Verlag KG Stuttgart · New York . ISSN 0032-0943

\section{Correspondence \\ Zhenjiang Li \\ Department of Nephrology \\ Shaanxi Provincial People's Hospital \\ 265 West Youyi Road \\ Xi'an, Shaanxi 710068 \\ P.R. China \\ lizhenjiang@medmail.com.cn}

\section{Abstract \\ $\nabla$}

Diabetic nephropathy is a progressive kidney disease caused by damage to the capillaries in the glomeruli. Endothelial dysfunction is an early sign of diabetic cardiovascular disease and may contribute to progressive diabetic nephropathy. Hyperglycemia-induced endothelial hyperpermeability is crucial to diabetic nephropathy. Rutin has beneficial effects on diabetic nephropathy, but the exact mechanisms of its protective effect remain elusive. The aim of this study was to assess the role of pretreatment with rutin in an in vitro model of hyperglycemia-induced barrier dysfunction in human renal glomerular endothelial cells. Human renal glomerular endothelial cells were exposed to rutin and/or hyperglycemia for $24 \mathrm{~h}$. Hyperglycemia increased permeability and decreased the junction protein occludin in the cellcell junction area and the total expression in human renal glomerular endothelial cells, whereas

\section{Introduction}

$\nabla$

The increase in the permeability of the glomerular barrier filtration to albumin is a well-known feature of diabetic microvasculature and a negative prognostic factor for vascular complications [1]. In patients with diabetes mellitus, microalbuminuria reflects a state of systemic endothelial dysfunction, also involving glomerular capillaries [2]. Hyperglycemia (HG) can rapidly increase systemic endothelial and glomerular barrier permeabilities in vivo. Such changes have also been amply demonstrated in the absence of insulin in endothelial monolayers in vitro [3-5]. Therefore, the endothelium plays an important role in the glomerular barrier upon HG $[1,6]$. By increasing the glomerular permeability, HG induces reactive oxygen species (ROS) production in endothelial cells and podocytes, as well as in mesangial cells. rutin treatment significantly corrected these abnormalities. Furthermore, hyperglycemiainduced activation of RhoA/ROCK was reversed by treatment with rutin or the knockdown of ROCK2. Interestingly, rutin prevented hyperglycemia-induced hyperpermeability, and dysfunction of the tight junction, a high level of reactive oxygen species, and activation of RhoA/ROCK were significantly abolished with the knockdown of Nrf2. In conclusion, rutin significantly prevented hyperglycemia-disrupted renal endothelial barrier function by inhibiting the RhoA/ROCK signaling pathway through decreasing reactive oxygen species, which was mediated by the activation of Nrf2. Our results may explain, at least in part, some beneficial effects of rutin that may be applicable to the treatment of vascular disorders in diabetic nephropathy.

Supporting information available online at http://www.thieme-connect.de/products 


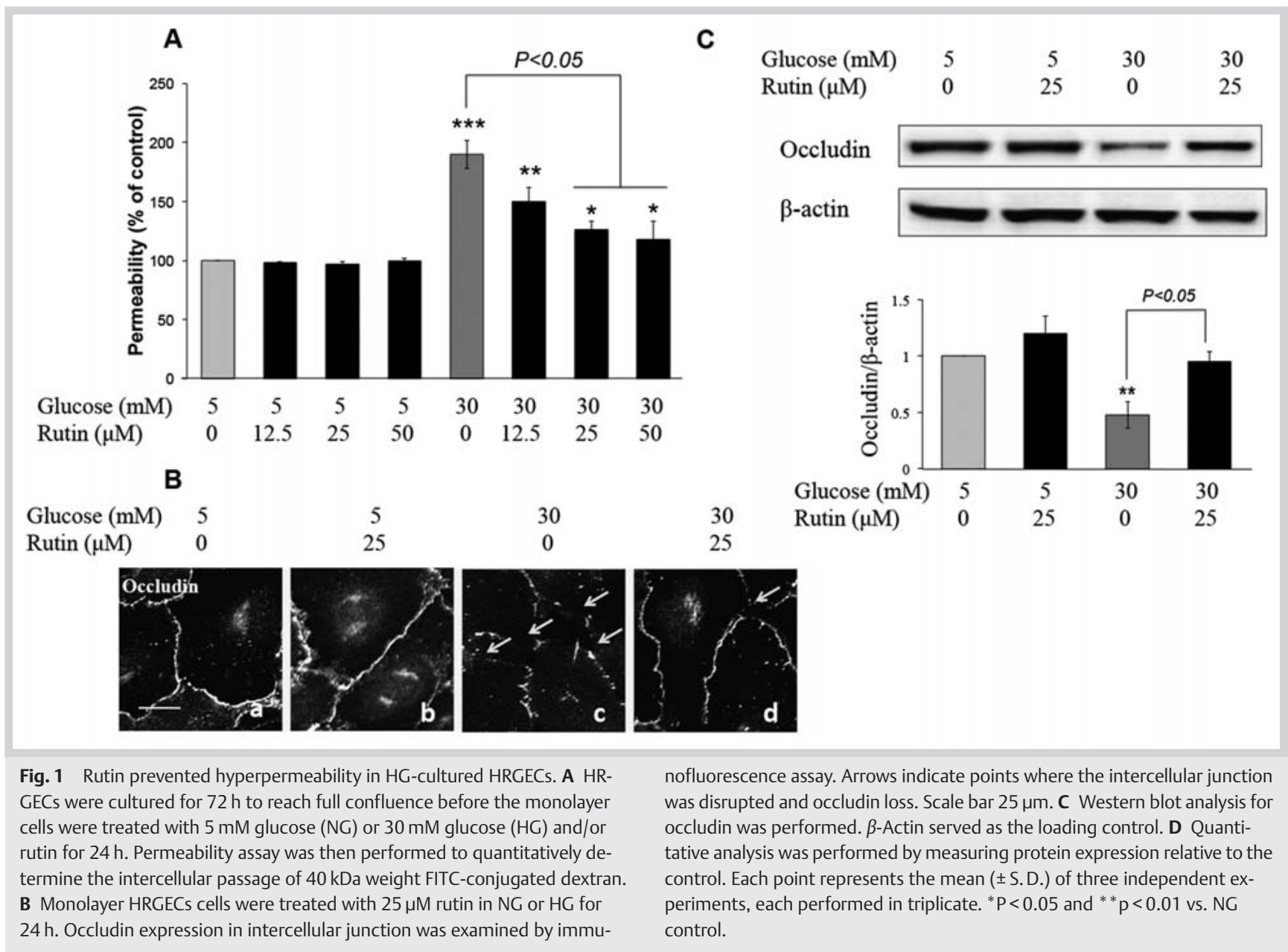

RhoA, a small GTPases of the Rho protein family, is a key regulator of the actin cytoskeleton [9]. Rho kinase (ROCK) is one of the first downstream targets of RhoA. RhoA/ROCK signaling regulates cell functions, including contraction [10], migration [11], adhesion [12], and cell cycle progression [13]. RhoA/ROCK signaling has recently attracted extensive attention as an important contributor to diabetic renal disease [14]. Activity of the RhoA/ROCK pathway is enhanced in vascular and renal cells in various types of kidney disorders. The factors involved in diabetes mellitus, such as high glucose, ROS, and the hexosamine pathway in vascular and renal cells, can activate the RhoA/ROCK signaling pathway $[15,16]$.

As a polyphenolic flavonoid, rutin has beneficial effects on renal damage by reducing oxidative stress $[17,18]$ with anticancer and anti-inflammatory activities as well. It has also been used to treat cardiovascular diseases and neurodegenerative disorders because of free radical-scavenging and antioxidant capacities [19]. Therefore, we hypothesized that rutin suppressed glomerular responses to HG in human renal glomerular endothelial cells (HRGECs). The aim of this study was to assess the role of rutin in an in vitro model of HG-induced barrier dysfunction in HRGECs.

\section{Results}

$\nabla$

The endothelial tight junction (TJ) regulates the permeability of endothelial cells, and exposure of monolayers of cultured renal endothelial cells to HG can significantly increase the permeability
$[20,21]$. In this study, HRGECs were treated with $12.5,25$, or $50 \mu \mathrm{M}$ rutin and/or HG for $24 \mathrm{~h}$. We detected HG-induced endothelial hyperpermeability by measuring the passage of FITC-dextran across the endothelium. As shown in $\odot$ Fig. 1, $24 \mathrm{~h}$ after incubation with HG, the endothelial permeability is obviously enhanced compared with that of cells treated with normal glucose (NG). However, treatment with rutin inhibited HG-induced endothelial barrier dysfunction ( $\boldsymbol{O}$ Fig. 1 A). Immunofluorescence staining and Western blotting ( $\odot$ Fig. 1 B, C) showed that $24 \mathrm{~h}$ of HG treatment significantly downregulated occludin expression in HRGECs. Furthermore, less occludin was distributed in the cell-cell junction area. Interestingly, the abnormal distribution and decreased expression of occludin were significantly reversed by treatment with rutin ( $\odot$ Fig. 1 B, C). Taken together, HG disrupted renal endothelial barrier function and rutin blocked this response in cultured HRGECS.

Small GTPases, especially RhoA and its downstream effector ROCK, regulated TJ formation, and HG can induce TJ dysfunction of glomerular endothelial cells through RhoA/ROCK signaling [5, 22]. $\odot$ Fig. 2 indicates that HG-treated HRGECs have significantly increased RhoA binding to GTP compared with that of NG-treated cells. ROCK was also activated because the phosphorylation rate of MYPT1 (a downstream effector of ROCK) was significantly increased by HG treatment ( $\boldsymbol{O}$ Fig. 2B). We then used siRNA to knockdown ROCK1 or ROCK2 or the inhibition of ROCK by using fasudil (ROCK inhibitor) in HRGECs. The results indicated that ROCK inhibition did not influence the expression of occludin 

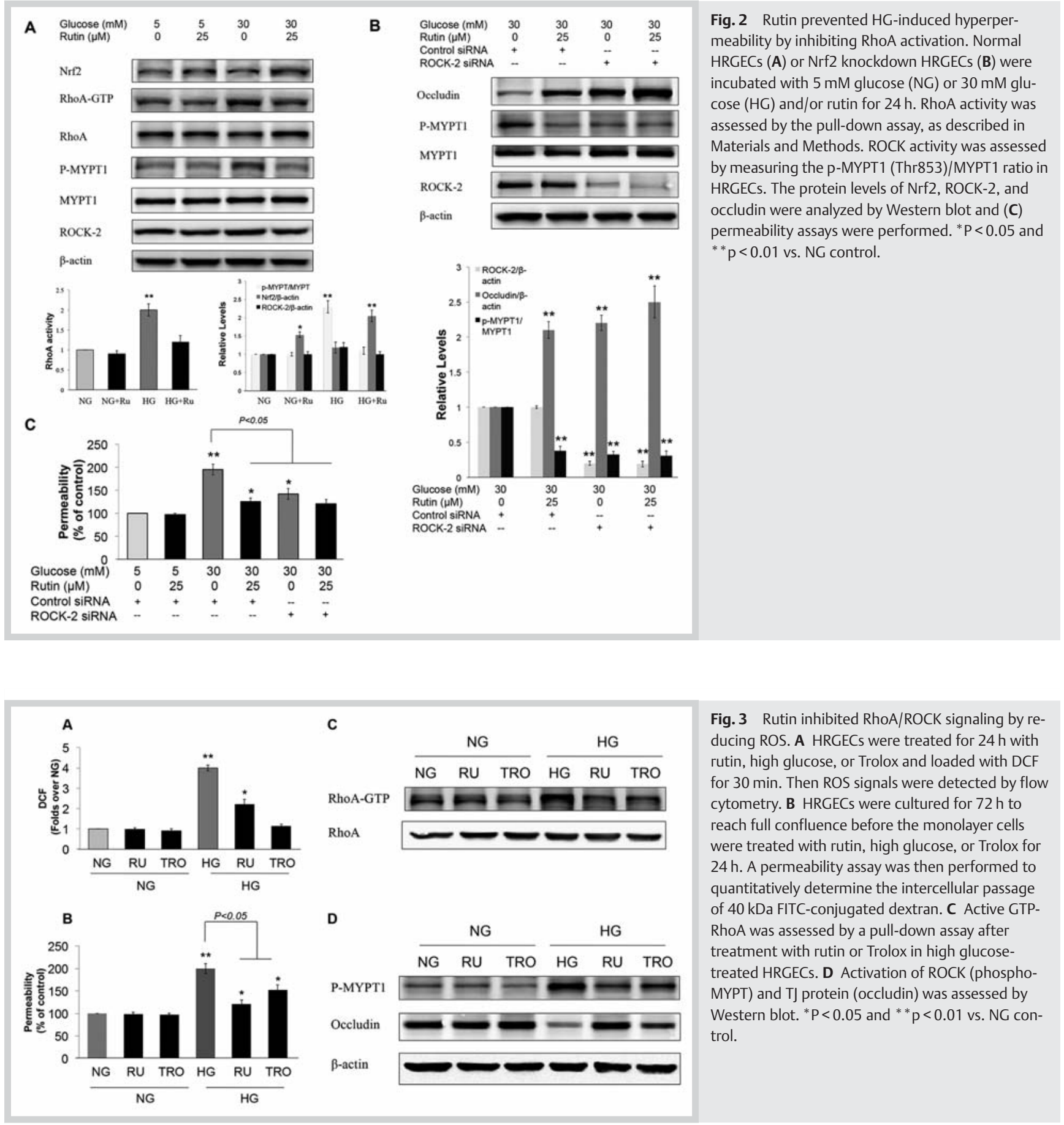

under NG conditions, but in the presence of HG, knockdown of ROCK or inhibition of ROCK activity, especially knockdown ROCK2, significantly increased occludin expression and decreased permeability ( $\odot$ Fig. $\mathbf{2 C}$ and Fig. S1, Supporting Information). Moreover, in the presence of $\mathrm{HG}$, treatment with rutin inhibited the activation of RhoA/ROCK, increased occludin expression, and decreased permeability. Therefore, rutin prevented HG-induced hyperpermeability through the inhibition of RhoA/ ROCK signaling.

Accumulating evidence has indicated that rutin was able to prevent vascular damage by exerting a direct antioxidant effect [2325]. In this study, we evaluated the inhibitory effect of rutin on HG-induced ROS generation in HRGECs. $\bullet$ Fig. 3 A shows that HG treatment led to significant ROS generation in HRGECs and rutin effectively abrogates HG-induced ROS generation. Trolox, a ROS scavenger, was used as a positive control ( $\bullet$ Fig. $3 \mathrm{~A}$ ). Thus, we assessed the effects of Trolox ( $1 \mathrm{mM})$ on RhoA activation. As shown in Fig. 3C, Trolox blocks HG-induced RhoA activation. However, Trolox did not affect the basal activity of RhoA or ROCK in HRGECs cultured in NG ( $<<0.05$; 0 Fig. $3 C)$. Furthermore, Trolox also blocked HG-induced hyperpermeability in HRGECs ( Fig. 3B, D). Thus, rutin inhibited RhoA/ROCK signaling by resisting oxidative stress.

Activation of the Nrf2 pathway attenuates HG-mediated high glucose-induced hyperpermeability by attenuating ROS production $[8,26]$. To clarify whether rutin prevented HG-disrupted renal 


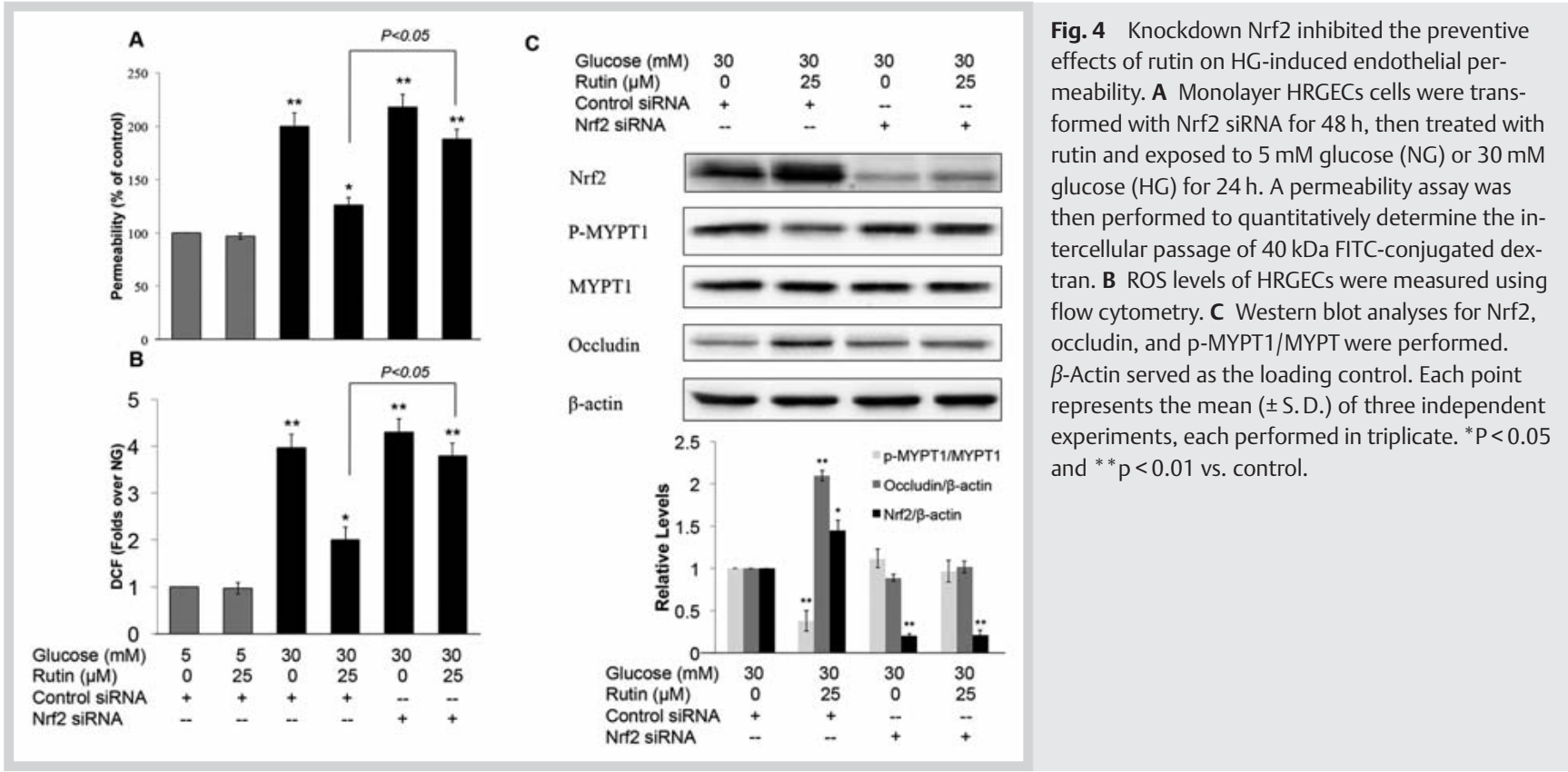

endothelial barrier function via the Nrf2 signaling pathway, HRGECs were transformed with Nrf2 siRNA for $48 \mathrm{~h}$. Rutin significantly increase nrf2 expression in $\mathrm{HG}$ and $\mathrm{NG}$ conditions ( Fig. 2A). Fig. 4 indicates that knockdown of Nrf2 significantly inhibits the preventive effects of rutin on HG-induced hyperpermeability ( Fig. 4A), ROS increase ( Fig.4B), activation of RhoA/ROCK, and occludin expression deficiency ( $\odot$ Fig.4C). Thus, rutin prevented HG-disrupted renal endothelial barrier function by activating Nrf2, which was mediated by decreasing ROS and then deactivating RhoA/ROCK.

\section{Discussion}

$\nabla$

The glomerular filtration barrier is a composite multilayered structure, and injury in one layer may spread to others and affect the whole function of the barrier [27]. It is well documented that endothelial glycocalyx plays a significant role in the glomerular filtration barrier $[27,28]$. Dysfunction of the endothelial TJ is a crucial step in the development of endothelial hyperpermeability that contributes to progressive DN [5]. HG is the main cause of all types of diabetic microvascular diseases. In addition, endothelial cell permeability is impaired by high concentrations of extracellular glucose upon diabetes mellitus [29,30]. It has previously been reported that rutin relieved renal damage by reducing oxidative stress $[17,18]$. However, the underlying mechanisms are still largely unexplored. In this study, HRGECs were exposed to rutin and/or HG for $24 \mathrm{~h}$. HG significantly increased the permeability of HRGECs. Moreover, the distribution of the junction protein occludin in the cell-cell junction area and the total expression in HRGECs were significantly decreased by HG treatment. However, treatment with rutin significantly reversed HGinduced endothelial dysfunctions, demonstrating that it prevented HG-induced endothelial permeability.

Activation of RhoA/ROCK signaling by HG disrupted the expression and translocation of occludin/ZO-1 and resulted in hyperpermeability [5]. In the presence of HG, treatment with rutin or knockdown of ROCK2 inhibited such activation, increased occlu- din expression, and decreased permeability. Therefore, rutin prevented HG-induced hyperpermeability through the inhibition of RhoA/ROCK signaling.

Oxidative stress is the most common disturbance resulting from HG. ROS generation is of particular importance in the alteration of endothelial permeability [2,26], and Nrf2 predominantly resists oxidative stress by decreasing ROS levels. Mounting evidence has indicated the potential role of Nrf2 in the protection and adaptation against HG-induced oxidative stress and inflammation $[8,26]$. Activation of the Nrf2 pathway attenuates HG-mediated high glucose-induced hyperpermeability by attenuating ROS production $[8,26]$. High glucose, ROS, and the hexosamine pathway in vascular and renal cells have been demonstrated to activate the RhoA/ROCK signaling pathway $[15,16]$.

Rutin herein prevented HG-induced hyperpermeability, and junction protein defects, activation of the RhoA/ROCK signaling pathway, and ROS were significantly abolished with the knockdown of Nrf2. Collectively, rutin managed to prevent HG-disrupted renal endothelial barrier function by inhibiting the RhoA/ROCK signaling pathway, which was mediated by decreasing ROS.

In conclusion, the ROS/RhoA/ROCK/occludin signaling pathway was involved in $\mathrm{HG}$-induced renal endothelial hyperpermeability. Since rutin inhibited the ROS/RhoA/ROCK signaling pathway by activating $\mathrm{Nrf2}$, this strategy may be applicable to the treatment of vascular disorders in DN.

\section{Material and Methods \\ $\nabla$}

\section{Chemicals}

Rutin ( $\geq 94 \%$ purity), Trolox, and DMSO were acquired from Sigma. Rutin was dissolved in DMSO to form a $100-\mathrm{mM}$ solution. Nrf2, Keap1, occludin, phospho-MYPT1/MYPT1, $\beta$-actin, antirabbit IgG $(\mathrm{H}+\mathrm{L}) 488$, and goat anti-rabbit IgG HRP-conjugated antibodies were all purchased from Cell Signaling Technology. Control siRNA, ROCK-2 siRNA, and Nrf2 siRNA were all purchased from Santa Cruz Biotechnology. 


\section{Cell culture and transfection}

HRGECS and CS-C complete medium (including $10 \%$ serum) or serum-free CS-C medium were purchased from Cell Systems. HRGECs were cultured in CS-C complete medium and antibiotics (100 IU $/ \mathrm{mL}$ penicillin and $100 \mu \mathrm{g} / \mathrm{mL}$ streptomycin). The cells were cultured in humidified air at $37^{\circ} \mathrm{C}$ with $5 \% \mathrm{CO}_{2}$, and then transfected with the indicated siRNA using Lipofectamine LTX (Life Technologies, Cat No.15338100) according to the manufacturer's protocol.

\section{Fluorescein isothiocyanate-dextran transwell assay}

Permeability across the HRGECs monolayer was studied in an in vitro vascular permeability system (Cat. No. ECM642), which has a $0.4-\mu \mathrm{m}$ pore size. HRGEC monolayers were plated on the insert of the transwell and cultured until confluence. After treatment, the same amount of FITC-dextran tracer ( $40 \mathrm{kDa}$, final concentration $20 \mu \mathrm{g} / \mathrm{mL}$ ) was added into the upper chamber and incubated for another $20 \mathrm{~min}$ at room temperature. The amount of tracer that penetrated the cell monolayer into the lower chamber was measured with the use of a fluorometer (Molecular Devices; excitation wavelength, $485 \mathrm{~nm}$; emission wavelength, $530 \mathrm{~nm}$ ) as the index of monolayer permeability of endothelial cells. The results are presented as fold change after correction with a blank control of NG. The data represent the means of three experiments.

\section{Immunofluorescence staining}

HRGECs were allowed to grow to confluence on fibronectincoated glass chamber slides. After treatment, the medium was aspirated and the monolayers were washed with PBS, fixed with $4 \%$ paraformaldehyde, and washed three times with PBS for $10 \mathrm{~min}$. Immunofluorescence staining was performed with a primary antibody against human occludin at a dilution of $1: 500$ overnight at $4^{\circ} \mathrm{C}$. The slides were photographed using an Olympus LCX100 Imaging System with an excitation wavelength of $546 \mathrm{~nm}$.

\section{Reactive oxygen species determination}

The levels of cellular superoxide were assessed by using DCFDA (Invitrogen) according to the manufacturer's instructions. Briefly, HRGECs were washed with phosphate-buffered saline and labeled at $37^{\circ} \mathrm{C}$ for $30 \mathrm{~min}$ in Hank's balanced salt solution (Gibco) containing $10 \mathrm{mM}$ DCFDA. Then the cells were trypsinized and resuspended in Hank's balanced salt solution. Fluorescence was measured by flow cytometry using a FACSCalibur flow cytometer (BD Biosciences).

\section{Western blot analysis}

After incubation with HG and/or rutin, cell monolayers were washed three times with PBS. The cells were scraped, harvested, and dissolved in gel buffer containing sodium dodecyl sulfate (SDS). Samples were loaded onto 5-20\% Super Sep SDS polyacrylamide gels (Wako Pure Chemical) and electrophoresed, and the proteins were transferred to polyvinylidenediuoride membranes (Millipore). The membranes were blocked in 5\% nonfat milk at room temperature for $1 \mathrm{~h}$, followed by overnight incubation at $4^{\circ} \mathrm{C}$ with primary antibodies with constant rotation. After incubation with secondary antibodies for $1 \mathrm{~h}$, proteins were detected using an enhanced ECL system (GE Healthcare). Semiquantifications were performed according to densitometric analysis in conjunction with the Quantity One software package. The signal of each band was normalized to that of actin in the same sample.
Small GTPase activity (small GTPase GTP-loading) assay Small GTPase GTP-loading assays were performed using the RhoA Activation Assay Biochem Kit (bead pull-down format) (Cytoskeleton). Briefly, the cell lysates were collected after treating HRGECs with HG and/or rutin. The GTP-bound small GTPases were captured by immobilized GST-tagged Rhotekin-RBD domain (Rho). After the beads were washed three times, the GTPbound small GTPases were released by boiling with Laemmli sample buffer at $95^{\circ} \mathrm{C}$ for $3-5 \mathrm{~min}$. Following standard Western blotting protocols as mentioned above, the GTP content in small GTPases was determined as the amount of Rho pulled down. GTP loading was expressed as the amount of GTP-bound small GTPase relative to that of total GTPase.

\section{Statistical analysis}

All experiments were repeated at least three times. All data are presented as means and standard deviations. Differences between the hyperpermeability of the control and treatment groups were determined with an independent samples t-test. The differences between groups were tested with a one-way ANOVA and Fisher's post hoc least significant difference test. All statistical tests were two-sided and a p value $<0.05$ was considered significant. All statistical analyses were performed with SPSS version 15.0 (SPSS).

\section{Supporting information}

Knockdown or inhibition of ROCK prevented HG-induced hyperpermeability.

\section{Conflict of Interest \\ $\nabla$}

We declare no conflict of interest.

References

1 Haraldsson B, Nystrom J. The glomerular endothelium: new insights on function and structure. Curr Opin Nephrol Hypertens 2012; 21: 258263

2 Sward P, Rippe B. Acute and sustained actions of hyperglycaemia on endothelial and glomerular barrier permeability. Acta Physiol (Oxf) 2012; 204: 294-307

3 Fu J, Lee K, Chuang PY, Liu Z, He JC. Glomerular endothelial cell injury and cross talk in diabetic kidney disease. Am J Physiol Renal Physiol 2015; 308: F287-F297

4 Luo P, Peng H, Li C, Ye Z, Tang H, Tang Y, Chen C, Lou T. Advanced glycation end products induce glomerular endothelial cell hyperpermeability by upregulating matrix metalloproteinase activity. Mol Med Rep 2015; 11: 4447-4453

5 Peng H, Luo P, Li Y, Wang C, Liu X, Ye Z, Li C, Lou T. Simvastatin alleviates hyperpermeability of glomerular endothelial cells in early-stage diabetic nephropathy by inhibition of RhoA/ROCK1. PLoS One 2013; 8: e80009

6 Friden $V$, Oveland E, Tenstad O, Ebefors $K$, Nystrom J, Nilsson UA, Haraldsson $B$. The glomerular endothelial cell coat is essential for glomerular filtration. Kidney Int 2011; 79: 1322-1330

7 Wang F, Pu C, Zhou P, Wang P, Liang D, Wang Q Hu Y, Li B, Hao X. Cinnamaldehyde prevents endothelial dysfunction induced by high glucose by activating Nrf2. Cell Physiol Biochem 2015; 36: 315-324

8 Wang C, Li C, Peng H, Ye Z, Zhang J, Liu X, Lou T. Activation of the Nrf2ARE pathway attenuates hyperglycemia-mediated injuries in mouse podocytes. Cell Physiol Biochem 2014; 34: 891-902

9 Sit ST, Manser E. Rho GTPases and their role in organizing the actin cytoskeleton. J Cell Sci 2011; 124: 679-683

10 Jiang HS, Zhu LL, Zhang Z, Chen H, Chen Y, Dai YT. Estradiol attenuates the TGF-beta1-induced conversion of primary TAFs into myofibroblasts and inhibits collagen production and myofibroblast contraction 
by modulating the Smad and Rho/ROCK signaling pathways. Int J Mol Med 2015; 36: 801-807

11 Li S, Dang Y, Zhou X, Huang B, Huang X, Zhang Z, Kwan YW, Chan SW, Leung GP, Lee SM, Hoi MP. Formononetin promotes angiogenesis through the estrogen receptor alpha-enhanced ROCK pathway. Sci Rep 2015; 5: 16815

12 Cheng L, Xu J, Qian YY, Pan HY, Yang H, Shao MY, Cheng R, Hu T. Interaction between mDia1 and ROCK in Rho-induced migration and adhesion of human dental pulp cells. Int Endod J 2015; DOI: 10.1111/ iej. 12587

13 Kumper S, Mardakheh FK, McCarthy A, Yeo M, Stamp GW, Paul A, Worboys J, Sadok A, Jorgensen C, Guichard S, Marshall CJ. Rho-associated kinase (ROCK) function is essential for cell cycle progression, senescence and tumorigenesis. Elife 2016; DOI: 10.7554/eLife.12203

14 Komers $R$. Rho kinase inhibition in diabetic kidney disease. Br J Clin Pharmacol 2013; 76: 551-559

15 Hirose A, Tanikawa T, Mori H, Okada Y, Tanaka Y. Advanced glycation end products increase endothelial permeability through the RAGE/ Rho signaling pathway. FEBS Lett 2010; 584: 61-66

16 Zhang Y, Peng F, Gao B, Ingram AJ, Krepinsky JC. Mechanical straininduced RhoA activation requires NADPH oxidase-mediated ROS generation in caveolae. Antioxid Redox Signal 2010; 13: 959-973

17 Kandemir FM, Ozkaraca M, Yildirim BA, Hanedan B, Kirbas A, Kilic K, Aktas E, Benzer $F$. Rutin attenuates gentamicin-induced renal damage by reducing oxidative stress, inflammation, apoptosis, and autophagy in rats. Ren Fail 2015; 37: 518-525

18 Han Y, Lu JS, Xu Y, Zhang L, Hong BF. Rutin ameliorates renal fibrosis and proteinuria in 5/6-nephrectomized rats by anti-oxidation and inhibiting activation of TGFbeta1-smad signaling. Int J Clin Exp Pathol 2015; 8: 4725-4734

19 Wagner C, Vargas AP, Roos DH, Morel AF, Farina M, Nogueira CW, Aschner M, Rocha JB. Comparative study of quercetin and its two glycoside derivatives quercitrin and rutin against methylmercury (MeHg)induced ROS production in rat brain slices. Arch Toxicol 2010; 84: 8997
20 Sajja RK, Green KN, Cucullo L. Altered Nrf2 signaling mediates hypoglycemia-induced blood-brain barrier endothelial dysfunction in vitro. PLoS One 2015; 10: e0122358

21 Clemmer JS, Xiang L, Lu S, Mittwede PN, Hester RL. Hyperglycemiamediated oxidative stress increases pulmonary vascular permeability. Microcirculation 2016; DOI: 10.1111/micc.12267

22 Ferrari A, Veligodskiy A, Berge U, Lucas MS, Kroschewski R. ROCK-mediated contractility, tight junctions and channels contribute to the conversion of a preapical patch into apical surface during isochoric lumen initiation. J Cell Sci 2008; 121: 3649-3663

23 Gong G, Qin Y, Huang W, Zhou S, Yang X, Li D. Rutin inhibits hydrogen peroxide-induced apoptosis through regulating reactive oxygen species mediated mitochondrial dysfunction pathway in human umbilical vein endothelial cells. Eur J Pharmacol 2010; 628: 27-35

24 Panchal SK, Poudyal H, Arumugam TV, Brown L. Rutin attenuates metabolic changes, nonalcoholic steatohepatitis, and cardiovascular remodeling in high-carbohydrate, high-fat diet-fed rats. J Nutr 2011; 141: 1062-1069

25 Ugusman A, Zakaria Z, Chua KH, Nordin NA, Abdullah Mahdy Z. Role of rutin on nitric oxide synthesis in human umbilical vein endothelial cells. ScientificWorldJournal 2014; 2014: 169370

26 Zuo X, Tian C, Zhao N, Ren W, Meng Y, Jin X, Zhang Y, Ding S, Ying C, Ye X. Tea polyphenols alleviate high fat and high glucose-induced endothelial hyperpermeability by attenuating ROS production via NADPH oxidase pathway. BMC Res Notes 2014; 7: 120

27 Maezawa Y, Takemoto M, Yokote K. Cell biology of diabetic nephropathy: Roles of endothelial cells, tubulointerstitial cells and podocytes. J Diabetes Investig 2015; 6: 3-15

28 Jeansson $M$, Bjorck $K$, Tenstad $O$, Haraldsson B. Adriamycin alters glomerular endothelium to induce proteinuria. J Am Soc Nephrol 2009, 20: $114-122$

29 Zhao XY, Wang XF, Li L, Zhang L, Shen DL, Li DH, Jin QS, Zhang JY. Effects of high glucose on human umbilical vein endothelial cell permeability and myosin light chain phosphorylation. Diabetol Metab Syndr 2015; 7: 98

30 Wardle EN. Vascular permeability in diabetics and implications for therapy. Diabetes Res Clin Pract 1994; 23: 135-139 